\title{
AS2077715 is a selective inhibitor of fungal mitochondrial cytochrome $b c_{1}$ complex
}

\author{
Keisuke Ohsumi, Masato Watanabe and Akihiko Fujie
}

AS2077715 is a novel antifungal metabolite produced by the newly isolated fungal strain Capnodium sp. 339855 . This compound has an analogous structure to funiculosin, an inhibitor of mitochondrial cytochrome $b c_{1}$ complex (complex III). AS2077715 inhibited ubiquinol-cytochrome $c$ reductase activity of Trichophyton mentagrophytes complex III with an IC $\mathrm{C}_{50}$ of $0.9 \mathrm{ng} \mathrm{ml}^{-1}$, while $6000-20000 \mathrm{ng} \mathrm{ml}^{-1}$ AS2077715 was required to obtain comparable inhibition of mammalian complex III. This inhibitor also suppressed the growth of $T$. mentagrophytes with a MIC of $0.08 \mu \mathrm{g} \mathrm{ml}^{-1}$, while cytotoxicity for mammalian cells was $>6 \mu \mathrm{g} \mathrm{ml}^{-1}$. These results indicate that AS2077715 is a selective inhibitor of fungal mitochondrial complex III. AS2077715 in doses of $1 \mu \mathrm{g} \mathrm{ml}^{-1}$ or greater showed fungicidal activity against $T$. mentagrophytes within $2 \mathrm{~h}$ of incubation. This early-onset effect of fungicidal activity was also exhibited by other complex III inhibitors. These results suggest that inhibition of complex III is a promising strategy for designing anti-Trichophyton agents and that AS2077715 can be a potential drug candidate for treating Trichophyton infections.

The Journal of Antibiotics (2014) 67, 713-716; doi:10.1038/ja.2014.67; published online 28 May 2014

\section{INTRODUCTION}

Onychomycosis, also known as nail fungal infection, is mainly caused by the dermatophytes Trichophyton rubrum and Trichophyton mentagrophytes. ${ }^{1-4}$ The introduction of oral antifungals, such as terbinafine and itraconazole, has improved the outcomes of treatment of onychomycosis, with clinical and mycological cure rates ranging from 70 to $90 \% .^{5}$ However, examinations carried out at considerably long follow-up intervals after completed therapy have revealed extremely high recurrence rates. ${ }^{6,7}$ Although the recurrence rates also include reinfection, it has been suggested that the fungi in infected nails cannot be eradicated completely because the antifungals used show no effect against the fungi in a dormant state, such as arthrospores, due to their mechanisms of action. ${ }^{8}$ Therefore, new antifungal agents that exhibit more potent fungicidal activity with unique modes of action are needed.

We previously reported the isolation of the novel antifungal compound AS2077715 (Figure 1) from the culture broth of Capnodium sp. 339855. This compound has an analogous structure to funiculosin (Figure 1), is an inhibitor of mitochondrial cytochrome $b c_{1}$ complex (complex III) with potent anti-Trichophyton activity. Almost all of the known complex III inhibitors, such as antimycin A, funiculosin and myxothiazol, possess antifungal activity. ${ }^{9}$ However, these inhibitors exhibit toxicity in mammals because they also inhibit mammalian complex III.

Here, we examine the inhibitory activity of AS2077715 against T. mentagrophytes complex III as compared with mammalian complex III. In addition, we examine the fungicidal activity of AS2077715 and some complex III inhibitors against T. mentagrophytes.

\section{MATERIALS AND METHODS}

\section{Compounds}

Funiculosin, ilicicolin $\mathrm{H}$ and myxothiazol were prepared in our laboratories. Antimycin A was purchased from Sigma-Aldrich Japan K.K. (Tokyo, Japan). Terbinafine was purchased from Tokyo Chemical Industry Co., Ltd. (Tokyo, Japan).

\section{Complex III inhibitory activity}

Complex III inhibitory activity was examined by an ubiquinol-cytochrome $c$ reductase assay using mitochondrial fractions obtained from T. mentagrophytes FP2103, rat liver and cultivated cells. Mitochondria fractions were prepared using a Mitochondria Isolation Kit for Tissue and Cultured Cells (BioChain Institute, Inc., Hayward, CA, USA) following the manufacturer's instructions. Briefly, a small piece of rat liver $(0.96 \mathrm{~g})$ was washed with phosphate-buffered saline and suspended in $10 \mathrm{ml}$ mitochondria isolation buffer, and then homogenized using a Polytron three times at 20000 r.p.m. for $20 \mathrm{~s}$. The homogenate was centrifuged at $600 \mathrm{~g}$ at $4^{\circ} \mathrm{C}$ for $10 \mathrm{~min}$, and the resulting supernatant was centrifuged at $12000 \mathrm{~g}$ at $4{ }^{\circ} \mathrm{C}$ for $15 \mathrm{~min}$. The pellet was suspended in mitochondria storage buffer for use as the sample and was stored at $-80^{\circ} \mathrm{C}$ until needed. Mitochondria fractions of T. mentagrophytes and cultivated cells were obtained from approximately $2 \times 10^{7}$ sonicated cells in same way described above. To initiate the assay, an aliquot of the mitochondria fractions diluted with assay buffer containing $50 \mathrm{~mm}$ potassium phosphate ( $\mathrm{pH} 7.0$ ), $250 \mathrm{~mm}$ sucrose, $0.2 \mathrm{~mm}$ EDTA, $1 \mathrm{~mm}$ sodium azide and $0.01 \%$ Tween- 20 were added to test compounds diluted with the same buffer. Potassium cyanide and cytochrome $c$ from bovine heart (Sigma-Aldrich Japan K.K.) were then added to final concentrations of $1 \mathrm{~mm}$ and $100 \mu \mathrm{g} \mathrm{ml}^{-1}$, respectively. The reaction was started by adding decylubiquinol, an ubiquinol analog, ${ }^{10}$ to a final concentration of $50 \mu \mathrm{M}$, and reduction of cytochrome $c$ was monitored at $550-539 \mathrm{~nm}$ with the SpectraMax spectrophotometer (Molecular Devices, Wokingham, UK). 
(1)<smiles>CCC(C)CC(C)CCC1CCCC(c2c(O)c(C3C(O)C(O)C(O)C3O)cn(C)c2=O)O1</smiles>

(2)<smiles>CC[C@H](C)C[C@@H](C)/C=C(\C)[C@H]1O[C@@H](c2c(O)c([C@@H]3[C@H](O)[C@H](O)[C@@H](O)[C@@H]3O)cn(C)c2=O)CC=C1C</smiles>

Figure 1 Chemical structures of AS2077715 (1) and funiculosin (2).

\section{Cell cytotoxicity assay}

RPMI 1640 medium (Invitrogen Japan, Tokyo, Japan) was used for the culture of Jurkat and EL4 cells. Primary human epidermal keratinocytes and human dermal fibroblast cells were cultured using HEK Growth Medium (Cell Applications, Inc., San Diego, CA, USA) and Growth Medium (Cell Applications, Inc.), respectively. Cytotoxicity was determined using the CellTiter-Blue Cell Viability Assay protocol (Promega KK, Tokyo, Japan) following the manufacturer's instructions. The cells were incubated for 3 days with each drug and the $50 \%$ inhibitory concentrations $\left(\mathrm{IC}_{50}\right)$ were determined by a nonlinear fit method using GraphPad Prism6 software (GraphPad Software, Inc., San Diego, CA, USA).

\section{ATP production inhibitory activity}

T. mentagrophytes FP2103 was cultured on a potato dextrose agar slant for 7 days at $30^{\circ} \mathrm{C}$, and spores were then harvested in sterile saline and filtered through gauze. Spores were precultured for $18 \mathrm{~h}$ at $37^{\circ} \mathrm{C}$ in Modified Sabouraud Dextrose medium, which was composed of $0.1 \%$ glucose, $0.2 \%$ Bacto tryptone, $0.2 \% \mathrm{~K}_{2} \mathrm{HPO}_{4}, 0.005 \% \mathrm{MgSO}_{4}$ and $0.005 \% \mathrm{CaCl}_{2}$, and incubated for $30 \mathrm{~min}$ at $37^{\circ} \mathrm{C}$ with each drug. ATP quantization in fungal cells was measured using the BacTiter-Glo Microbial Cell Viability Assay (Promega KK) in accordance with the manufacturer's instructions.

\section{Time-kill curve assay}

Test compounds were dissolved in dimethyl sulfoxide and added to $1.5 \mathrm{ml}$ tubes in $10 \mu \mathrm{l}$ aliquots. Spores $\left(1 \times 10^{5}\right)$ of T. mentagrophytes in Modified Sabouraud Dextrose medium $(990 \mu \mathrm{l})$ were added to each tube, and the tubes were then incubated for $48 \mathrm{~h}$ without shaking at $30^{\circ} \mathrm{C}$ for active phase, or $4{ }^{\circ} \mathrm{C}$ for dormant phase. To test the fungicidal effects to germinating spores, spores of T. mentagrophytes that had been incubated for $18 \mathrm{~h}$ were added to each tube containing test compounds. At several time points, colony formation assays were performed. Briefly, $50 \mu \mathrm{l}$ aliquots of the test tube were transferred to a $10-\mathrm{cm}$ dish, into which $20 \mathrm{ml}$ Sabouraud Dextrose agar medium was added, after being melted and cooled to $40{ }^{\circ} \mathrm{C}$. The dish was incubated for $24 \mathrm{~h}$ at $30^{\circ} \mathrm{C}$, and the colony-forming units per dish were counted. The number of colony-forming units at $0 \mathrm{~h}$ of a control sample was adopted as that at $0 \mathrm{~h}$ of test compounds.

\section{RESULTS}

Complex III inhibitory activity of AS2077715 against

T. mentagrophytes and mammalian cells

Funiculosin (Figure 1b), a nonselective inhibitor of complex III, showed potent inhibitory activity against T. mentagrophytes a
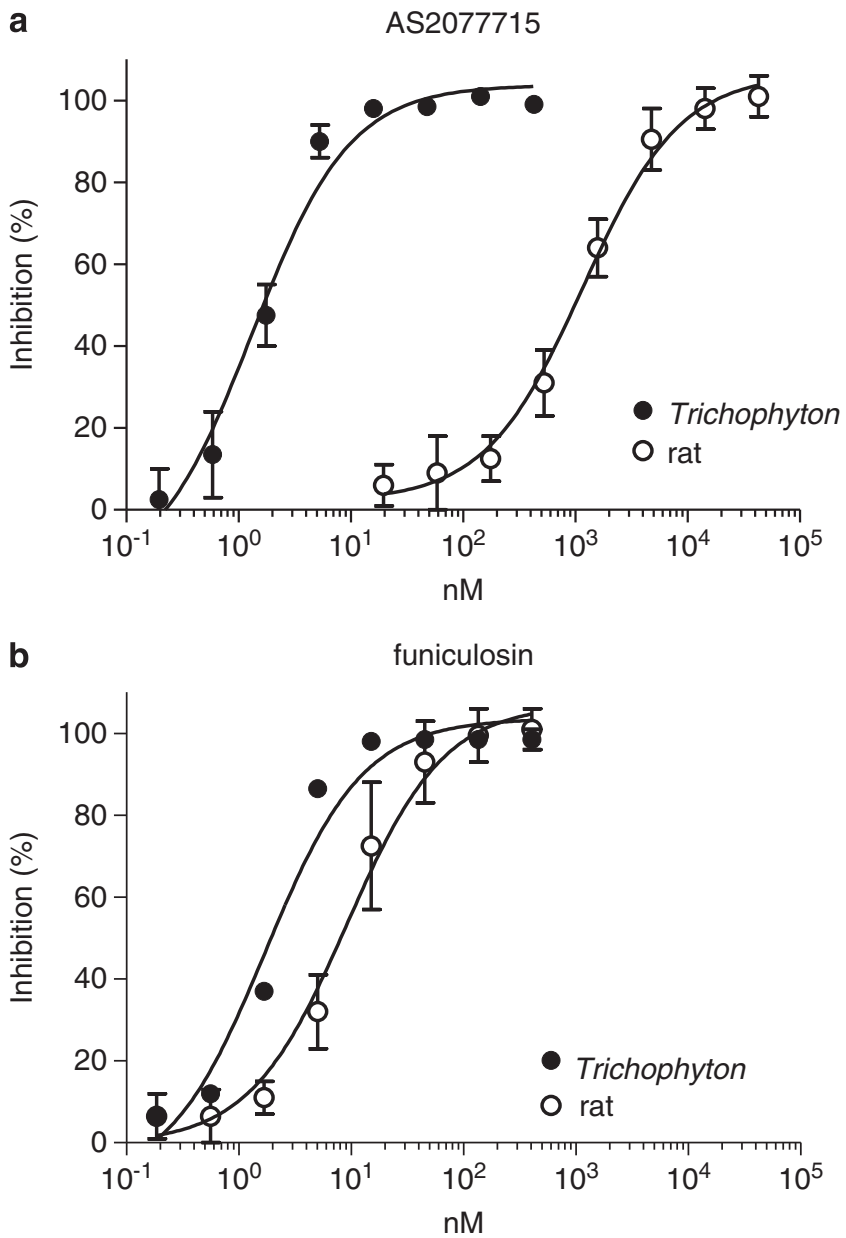

Figure 2 Complex III inhibitory activity of AS2077715 against T. mentagrophytes and rat.

Table 1 Complex III inhibitory activity and cytotoxicity of AS2077715, funiculosin and antimycin A against T. mentagrophytes and mammalian cells

$\mathrm{IC}_{50}\left(\mathrm{ngml}^{-1}\right)$

(Cytotoxicity:complex III inhibition)

\begin{tabular}{lccc} 
Cell type & AS2077715 & Funiculosin & Antimycin A \\
\hline Trichophyton mentagrophytes & $80: 0.9$ & $70: 1.5$ & $1300: 0.4$ \\
EL4 (mouse) & $6200: 480$ & $35: 1.9$ & $5.4: 0.28$ \\
Jurkat (Human) & $20000: 860$ & $1400: 180$ & $1.6: 0.12$ \\
Human epidermal keratinocytes & $6000: 500$ & $120^{\mathrm{a}}: 19$ & $0.8^{\mathrm{a}}: 0.30$ \\
Human dermal fibroblasts & $11000: 690$ & $490^{\mathrm{a}}: 41$ & $6.3^{\mathrm{a}}: 0.37$ \\
\hline
\end{tabular}

$\mathrm{al}_{30}\left(\mathrm{ng} \mathrm{ml}^{-1}\right)$

$\left(\mathrm{IC}_{50}: 1.7 \mathrm{~nm}\right)$ and rat complex III $\left(\mathrm{IC}_{50}: 9.2 \mathrm{~nm}\right)$ (Figure 2b). AS2077715 (Figure 1a) exhibited potent inhibitory activity against complex III of T. mentagrophytes ( $\mathrm{IC}_{50}: 1.4 \mathrm{~nm}$ ) (Figure $2 \mathrm{a}$ ), but had 860 -fold weaker activity against rat complex III ( $\mathrm{IC}_{50}$ : $\left.1200 \mathrm{~nm}\right)$ compared with funiculosin. The cytotoxicity and complex III inhibitory activities of AS2077715 and other complex III inhibitors against mouse and human cells are shown in Table 1 . The $\mathrm{IC}_{50}$ for 


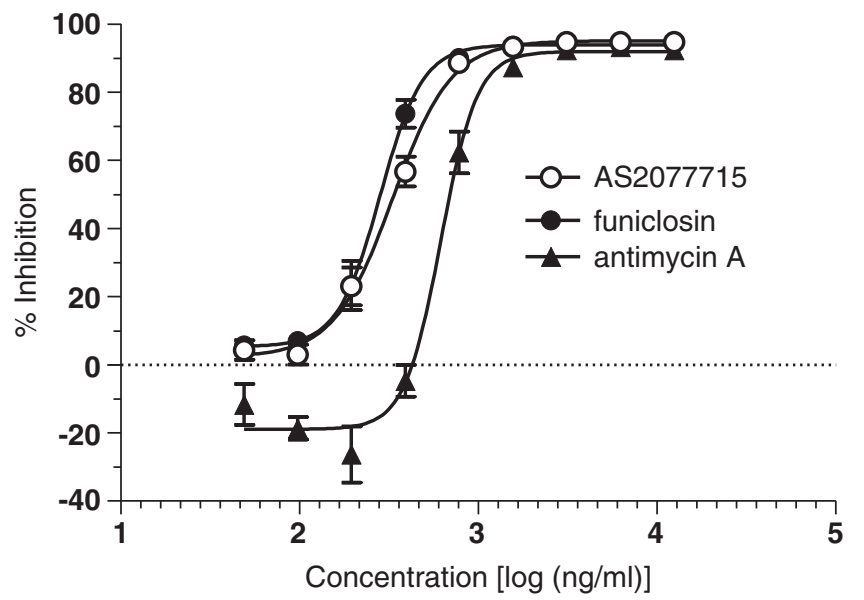

Figure 3 Inhibitory activities of AS2077715, funiculosin and antimycin A against ATP production in T. mentagrophytes.
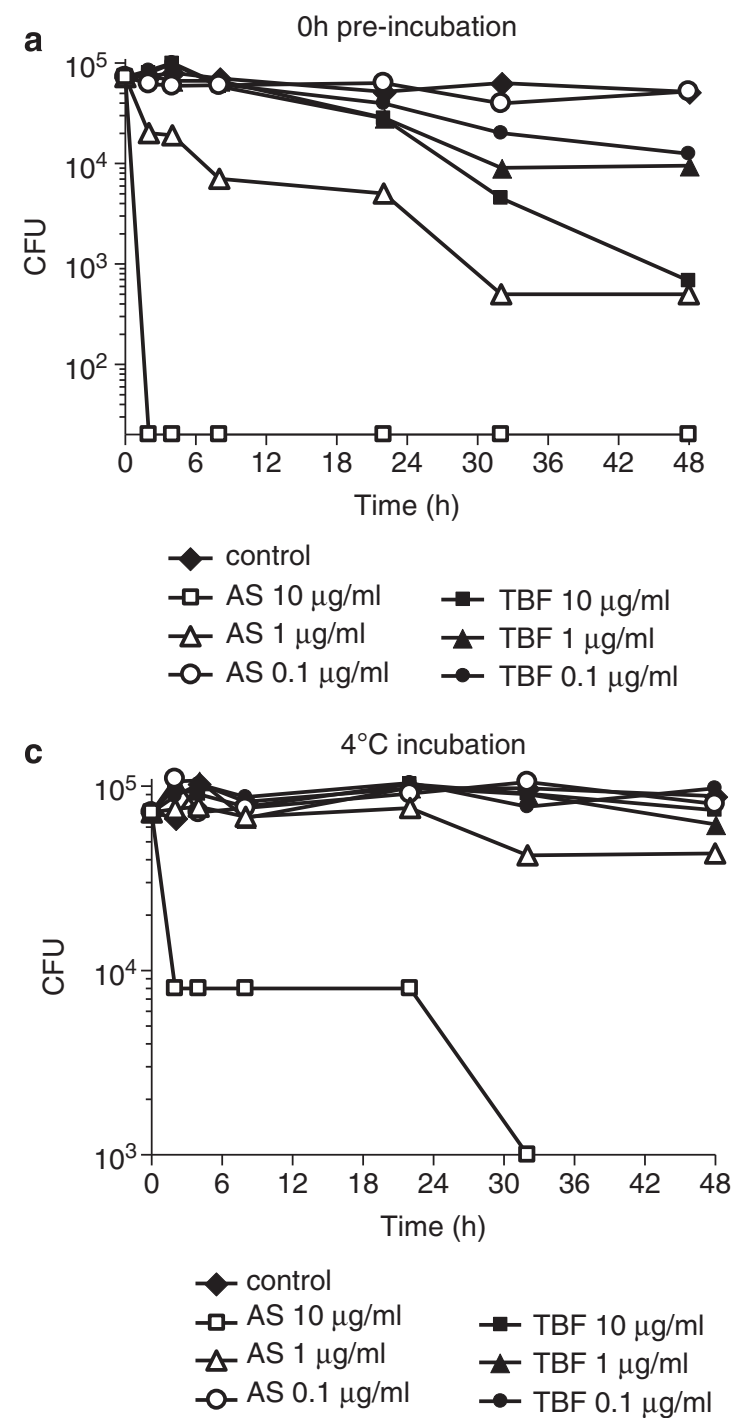

antimycin A, a nonselective inhibitor of complex III, was similar for mouse cells, human cells and T. mentagrophytes. Funiculosin showed more than 10-fold weaker inhibitory activity against human complex III than against complex III in T. mentagrophytes. The complex III inhibitory activities of AS2077715 in mouse and human cells were more than 500-fold weaker than the inhibitory activity in T. mentagrophytes. Thus, AS2077715 showed highly selective inhibitory activity against $T$. mentagrophytes complex III.

The cytotoxicity of these inhibitors for mouse and human cells was correlated with the complex III inhibitory activities. The cytotoxicity of AS2077715 in mouse and human cells was more than 70 times weaker than that in T. mentagrophytes.

\section{Inhibition of fungal ATP production}

The inhibitory activity of AS2077715 against ATP production of T. mentagrophytes was evaluated by quantitation of the ATP present in fungal cells (Figure 3). AS2077715 showed ATP reduction activity
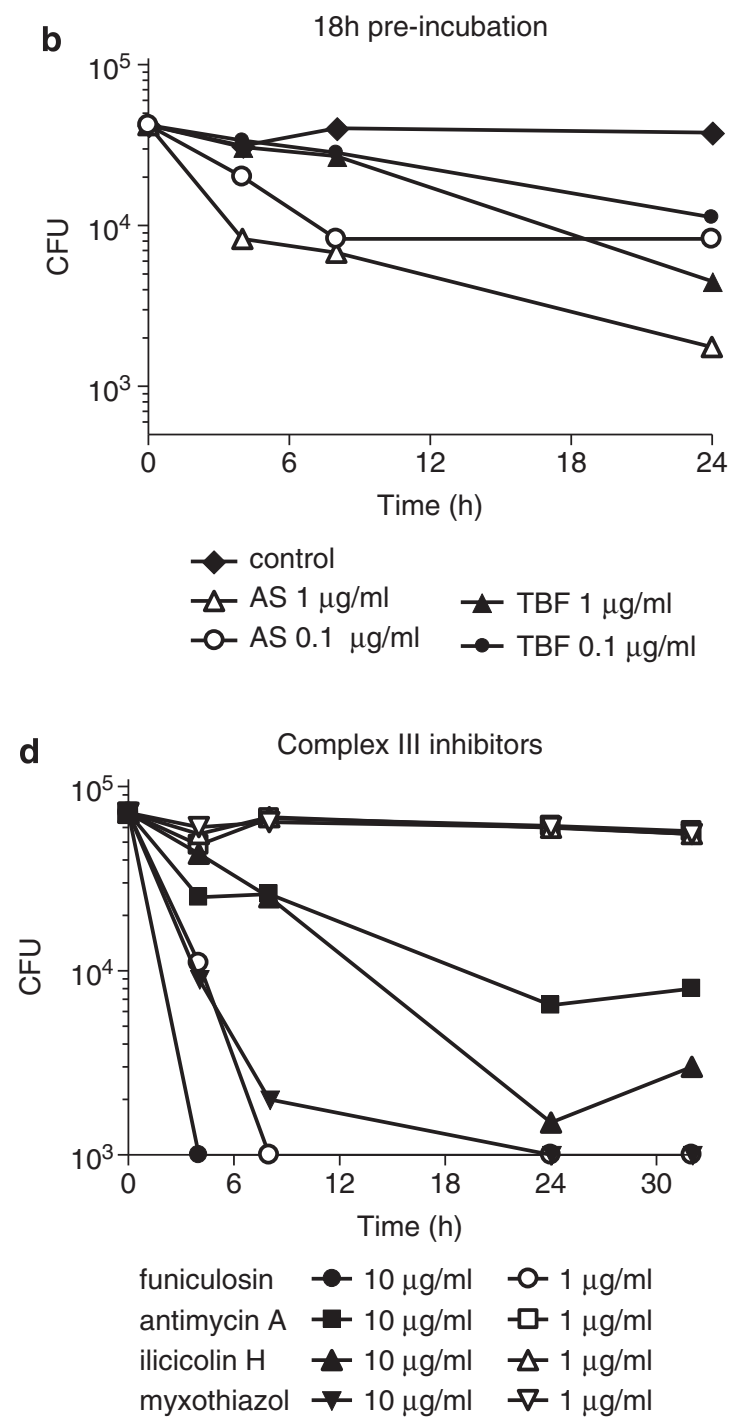

Figure 4 Time-kill curve plots in T. mentagrophytes. (a) Test compounds and spores of $T$. mentagrophytes in medium were mixed and incubated at $30^{\circ} \mathrm{C}$; (b) Test compounds and 18-h incubated spores of T. mentagrophytes in medium were mixed and incubated at $30^{\circ} \mathrm{C}$; (c) same as a except $4{ }^{\circ} \mathrm{C}$ incubation; (d) same as a. 
against $T$. mentagrophytes with an $\mathrm{IC}_{50}$ value of $0.33 \mu \mathrm{g} \mathrm{ml}^{-1}$. The $\mathrm{IC}_{50}$ values of funiculosin and antimycin A were $0.28 \mu \mathrm{g} \mathrm{ml}^{-1}$ and $0.62 \mu \mathrm{g} \mathrm{ml}^{-1}$, respectively. AS2077715 and other complex III inhibitors showed 95\% reduction of ATP level in fungal cells within $30 \mathrm{~min}$ of incubation.

\section{Time-kill curve assay}

Terbinafine showed fungicidal activity against T. mentagrophytes at $>22 \mathrm{~h}$ after administration, whereas 1 and $10 \mu \mathrm{g} \mathrm{ml}^{-1}$ of AS2077715 showed fungicidal activity within $2 \mathrm{~h}$ of incubation (Figure $4 \mathrm{a}$ ). To test fungicidal effects on germinating spores, test compounds were added to media containing spores which were incubated for $18 \mathrm{~h}$ (Figure $4 \mathrm{~b}$ ). Terbinafine showed fungicidal activity against germinating spores from 8 to $24 \mathrm{~h}$ after administration, whereas $1 \mu \mathrm{g} \mathrm{ml}^{-1}$ of AS2077715 showed fungicidal activity within $4 \mathrm{~h}$ of incubation (Figure 4b). Thus, AS2077715 exhibited earlier onset of fungicidal activity than terbinafine. The onset of fungicidal activity within several hours suggested that AS2077715 is able to effect to the spores in their resting state. Therefore, we evaluated the fungicidal activities of AS2077715 at $4{ }^{\circ} \mathrm{C}$ incubation. Ten $\mu \mathrm{g} \mathrm{ml}^{-1}$ of terbinafine did not show fungicidal activity after incubation at $4^{\circ} \mathrm{C}$ for $48 \mathrm{~h}$, whereas $10 \mu \mathrm{g} \mathrm{ml}^{-1}$ of AS2077715 showed fungicidal activity after incubation at $4{ }^{\circ} \mathrm{C}$ for $2 \mathrm{~h}$ (Figure $4 \mathrm{c}$ ). From this result, we concluded that the drug was active against resting spores. Next, to clarify whether this early onset of fungicidal activity was a specific characteristic of AS2077715 or a general characteristic of complex III inhibitors, we evaluated the fungicidal activities of other complex III inhibitors. All of the tested complex III inhibitors showed fungicidal activities within $8 \mathrm{~h}$ (Figure 4d). Therefore, the early onset of fungicidal activities of AS2077715 against T. mentagrophytes was deemed to be due to inhibition of complex III.

\section{DISCUSSION}

From the structural similarity to funiculosin, AS2077715 was expected to be an inhibitor of complex III, and AS2077715 was found to inhibit the complex III activities of T mentagrophytes (Figure 2) and reduce the intracellular ATP content of T mentagrophytes at the same concentrations as funiculosin (Figure 3). In contrast, concentrations of AS2077715 ranging from 14 to 250 times those of funiculosin were required to inhibit mammalian complex III activities (Figure 2 and Table 1). These results suggest that AS2077715 is a highly selective inhibitor against T. mentagrophytes complex III, with reduced cytotoxicity in mammalian cells compared with funiculosin (Table 1).

Because funiculosin inhibits at the ubiquinone reduction site (Qn site) of cytochrome $b,{ }^{10}$ AS2077715 probably also inhibits at the Qn site. Ilicicolin $\mathrm{H}$, another Qn site inhibitor, also shows selectivity to yeast complex III compared with bovine heart complex III. ${ }^{11}$ These differences are thought to result from subtle structural differences in the Qn pocket in fungi and mammalian enzymes. The amino acid residues likely responsible for this difference are probably some of those that confer resistance to funiculosin. ${ }^{12,13}$ However, a deeper understanding of the structural basis for the species specificity requires a crystallographic analysis of the structure of the complex III with AS2077715 bound. The existence of selective inhibitors of fungal complex III, including AS2077715, suggests that it should be possible to design novel inhibitors targeting complex III with appropriate selectivity to allow their use as drugs against pathogenic fungi.
AS2077715 showed fungicidal efficacy against both dormant and proliferating T. mentagrophytes within several hours of incubation (Figures $4 \mathrm{a}-\mathrm{c}$ ). The time required for this compound to exhibit a fungicidal effect was shorter than that of terbinafine (Figure $4 \mathrm{~b}$ ), and terbinafine did not exhibit fungicidal activity against dormant T. mentagrophytes at the tested concentrations (Figure 4c). All azoles, terbinafine and amorolfine exert their principal action mainly against proliferating organisms, which synthesize ergosterol for the production of new cell membranes. The subungual tissue of fungally infected nails, however, is regularly found to contain arthrospores that do not proliferate and cannot be killed by these antifungals. Successful treatment of onychomycosis in the infection site depends not only on achieving the MIC of the antifungal agent-usually determined on fresh, proliferating fungal strains - but also on the efficacy against fungal spores dormant in nail keratin.

The early onset of fungicidal activities of AS2077715 was a common effect in complex III inhibitors (Figure 4d). Reactive oxygen species generation due to the blockade of electron flow at complex III is known to inhibit the growth of S. cerevisiae. ${ }^{14}$ The mechanism of early onset by complex III inhibitors remains to be examined.

In conclusion, selective inhibition of complex III is a promising strategy to design anti-Trichophyton agents, and AS2077715 is a potential drug candidate for treating Trichophyton infections. We plan to evaluate the in vivo activities of AS2077715 against T. mentagrophytes, and will describe the findings in a subsequent paper.

1 Faergemann, J. \& Baran, R. Epidemiology, clinical presentation and diagnosis of onychomycosis. Br. J. Dermatol. 149 (Suppl 65), 1-4 (2003).

2 Foster, K. W., Ghannoum, M. A. \& Elewski, B. E. Epidemiologic surveillance of cutaneous fungal infection in the United States from 1999 to 2002. J. Am. Acad. Dermatol. 50, 748-752 (2004).

3 Ghannoum, M. A. et al. A large-scale North American study of fungal isolates from nails: the frequency of onychomycosis, fungal distribution, and antifungal susceptibility patterns. J. Am. Acad. Dermatol. 43, 641-648 (2000).

4 Kaur, R., Kashyap, B. \& Bhalla, P. Onychomycosis-epidemiology, diagnosis and management. Indian. J. Med. Microbiol. 26, 108-116 (2008).

5 Wenig, J. A. The systemic treatment of onychomycosis. Clin. Podiatr. Med. Surg. 21, 579-589, vi (2004).

6 Piraccini, B. M., Sisti, A. \& Tosti, A. Long-term follow-up of toenail onychomycosis caused by dermatophytes after successful treatment with systemic antifungal agents. J. Am. Acad. Dermatol. 62, 411-414 (2010).

7 Gupta, A. K., Cooper, E. A. \& Paquet, M. Recurrences of dermatophyte toenail onychomycosis during long-term follow-up after successful treatments with mono- and combined therapy of terbinafine and itraconazole. J. Cutan. Med. Surg. 17, 201-206 (2013).

8 Seebacher, C. Action mechanisms of modern antifungal agents and resulting problems in the management of onychomycosis. Mycoses 46, 506-510 (2003).

9 Masuma, R., Shiomi, K. \& Ōmura, S. in The Mycota Vol. 15 (eds Anke, T \& Weber, D) Ch. 12, 247-271 (Springer, Berlin Heidelberg, 2009).

10 von Jagow, G. \& Link, T. A. Use of specific inhibitors on the mitochondrial bc 1 complex. Methods Enzymol. 126, 253-271 (1986).

11 Gutierrez-Cirlos, E. B., Merbitz-Zahradnik, T. \& Trumpower, B. L. Inhibition of the yeast cytochrome bc1 complex by ilicicolin $\mathrm{H}$, a novel inhibitor that acts at the $\mathrm{Qn}$ site of the bc1 complex. J. Biol. Chem. 279, 8708-8714 (2004).

12 di Rago, J. P., Perea, J. \& Colson, A. M. Isolation and RNA sequence analysis of cytochrome $b$ mutants resistant to funiculosin, a center i inhibitor of the mitochondrial ubiquinol-cytochrome c reductase in Saccharomyces cerevisiae. FEBS Lett. 263, 93-98 (1990).

13 Brasseur, G. \& Brivet-Chevillotte, P. Specificities of the two center N inhibitors of mitochondial bc1 complex, antimycin and funiculosin: strong involvement of cytochrome b-asparagine-208 in funiculosin binding. FEBS Lett. 354, 23-29 (1994).

14 Fujita, K., Tani, K., Usuki, Y., Tanaka, T. \& Taniguchi, M. Growth inhibition dependent on reactive oxygen species generated by C9-UK-2A, a derivative of the antifungal antibiotic UK-2A, in Saccharomyces cerevisiae. J. Antibiot. 57, 511-517 (2004). 